\title{
Postsurgical Meningitis Complicated by Severe Refractory Intracranial Hypertension with Limited Treatment Options: The Role of Mild Therapeutic Hypothermia
}

\author{
Konstantin A. Popugaev ${ }^{1}$ Ivan A. Savin ${ }^{1}$ Andrew V. Oshorov ${ }^{1}$ Natalia V. Kurdumova ${ }^{1}$ \\ Olga N. Ershova ${ }^{1}$ Andrew U. Lubnin ${ }^{2}$ Boris A. Kadashev ${ }^{3}$ Pavel L. Kalinin ${ }^{3}$ Maxim A. Kutin ${ }^{3}$ \\ Tim Killeen ${ }^{4}$ Evaldas Cesnulis ${ }^{4}$ Ronald Melieste ${ }^{5}$
}

\footnotetext{
${ }^{1}$ Neurocritical Care Department, Burdenko Neurosurgical Research Institute, Russian Academy of Medical Sciences, Moscow, Russian Federation

2 Department of Neuroanesthesia, Burdenko Neurosurgical Research Institute, Russian Academy of Medical Sciences, Moscow, Russian Federation

3 8th Neurosurgical Department, Burdenko Neurosurgical Research Institute, Russian Academy of Medical Sciences, Moscow, Russian Federation

${ }^{4}$ Department of Neurosurgery, Klinik Hirslanden, Zürich, Switzerland

${ }^{5}$ Temperature Management Division Europe, Zoll Medical

Corporation, Chelmsford, Massachusetts, United States
}

Address for correspondence K. Popugaev, MD, PhD, Burdenko Neurological Research Institute, 4th Tverskaya-Yamskaya 16, Moscow, Russian Federation (e-mail: stan.popugaev@yahoo.com).

J Neurol Surg Rep 2014;75:e224-e229.

\begin{abstract}
Intracranial hypertension is a commonly encountered neurocritical care problem. If firsttier therapy is ineffective, second-tier therapy must be initiated. In many cases, the full arsenal of established treatment options is available. However, situations occasionally arise in which only a narrow range of options is available to neurointensivists. We present a rare clinical scenario in which therapeutic hypothermia was the only available method for controlling intracranial pressure and that demonstrates the efficacy and safety of the Thermogard (Zoll, Chelmsford, Massachusetts, United States) cooling system in creating and maintaining a prolonged hypothermic state. The lifesaving effect of hypothermia was overshadowed by the unfavorable neurologic outcome observed (minimally conscious state on intensive care unit discharge). These results add further evidence to support the role of therapeutic hypothermia in managing intracranial pressure and provide motivation for finding new strategies in combination with hypothermia to improve neurologic outcomes.
\end{abstract}

\section{Introduction}

Intracranial hypertension (ICH) is one of the fundamental problems in neurocritical care. The Columbia stepwise protocol for intracranial pressure (ICP) management is generally accepted. ${ }^{1}$ When first-tier therapies-ventricular drainage, sedation, cerebral perfusion pressure optimization, and osmotherapy-fail, second-tier therapy must be initiated. Second-tier options comprise hyperventilation, decompressive hemicraniectomy, barbiturate coma, and therapeutic received

March 20, 2014

accepted

June 3, 2014

published online

August 21, 2014
DOI http://dx.doi.org/

10.1055/s-0034-1387188. ISSN 2193-6358. (c) 2014 Georg Thieme Verlag KG
Stuttgart · New York

License terms

(c) $(1) \$$ 


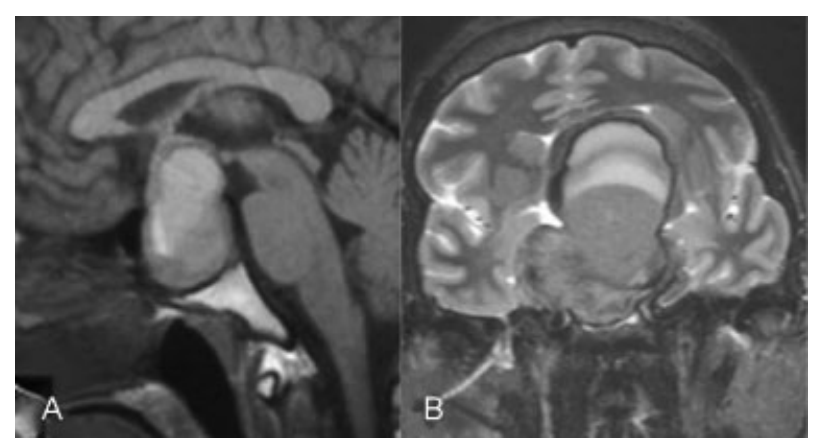

Fig. 1 (A) Sagittal T1-weighted magnetic resonance imaging (MRI) showing large sellar mass and associated hemorrhage. (B) Coronal T2weighted MRI of the same lesion.

hypothermia. Intensivists and neurosurgeons often pursue a strategy involving a combination of second-tier ICP correction methods, but occasionally only one of these methods is available. We present such a case in which mild therapeutic hypothermia proved lifesaving as the only available alternative.

\section{Case Report}

A 35-year-old white man presented with obtundation, right hemiparesis, headache, nausea, vomiting, and acute bilateral blindness. He initially presented to a peripheral hospital where magnetic resonance imaging (MRI) showed a sellar region tumor with evidence of tumor hemorrhage (-Fig. $\mathbf{1}$ ).

The patient was transferred to our institution (a tertiary referral center) with a diagnosis of pituitary apoplexy. On arrival the patient's Glasgow Coma Scale (GCS) was 9/15 (E1V3M5) with episodes of stereotypic movements. There was a marked right hemiparesis with a Medical Research Council grade $3 / 5$ weakness of the right arm proximally and distally and 0/5 weakness in the right leg. The left side was normal. Visual examination demonstrated a bilateral amaurosis with right abducens and bilateral oculomotor palsies. Panhypopituitarism was evidenced by bradycardia (50-55 bpm), reduced intestinal peristalsis, mild hyponatremia (129-132 mmol/L), and low levels of circulating adrenocorticoid, thyroid, and gonadotropic hormones and insulinlike growth factor 1 . Blood pressure (BP) was normal $(120 / 80 \mathrm{~mm} \mathrm{Hg})$, as was his respiratory rate (RR). Treatment with hydrocortisone (400 mg/day intravenously) and levo- thyroxine ( $3 \mu \mathrm{g} / \mathrm{kg} / \mathrm{day}$ via nasogastric tube) was started immediately.

The following day, an endoscopic transsphenoidal removal of the tumor and attendant blood clots was successfully performed (-Fig. 2). An external lumbar drain was inserted immediately prior to the procedure. A further drain was inserted into the tumor cavity following resection for postoperative hemorrhage control. Histologic examination excluded pituitary adenoma and instead confirmed a diagnosis of pilocytic astrocytoma. The patient was successfully extubated in the intensive care unit (ICU) 3 hours postoperatively. The right hemiparesis and visual loss were unchanged.

The patient remained in the ICU for 12 days. Lumbar drainage was removed on the second postoperative day; the tumor cavity drain was removed on day 5. GCS improved from 9/15 (E1V3M5) at extubation to 12/15 (E1V5M6) on day 5. Motor function in the right limbs also improved to $5 / 5$ in the right arm and $4 / 5$ in the right leg. On the left, visual acuity improved to 20/40 and cranial nerve deficits resolved, although the visual fields were narrowed. There was no visual function in the right eye, and the right abducens palsy remained. Vital signs were stable (BP: 120/80-130/80 mm $\mathrm{Hg}$; heart rate [HR]: 55-65 bpm, RR: 12-15/min), and electrolyte disturbances were corrected $\left(\mathrm{Na}^{+}: 135-140 \mathrm{mmol} / \mathrm{L}\right)$.

The patient was discharged on day 12 to the neurosurgical ward where rehabilitation continued and the patient was able to walk with assistance. Sudden cerebrospinal fluid (CSF) rhinorrhea occurred when the patient blew his nose on the postoperative day 15. A combined intranasal spray (polydexa, comprising neomycin, polymyxin $B$, dexamethasone, and phenylephrine) was administered. The next day, the patient experienced a generalized seizure despite prophylactic antiepileptic valproate therapy. Seizure activity was terminated with benzodiazepines, but the patient's GCS was 8/15 (E1V2M5), and he was intubated, ventilated, and returned to the ICU. Brain computed tomography (CT) showed pneumocephalus (-Fig. 3).

The patient remained comatose despite the absence of epileptiform activity on continuous electroencephalograph monitoring and treatment with intravenous valproate and levetiracetam. Postoperative meningitis was suspected, and broad-spectrum antimicrobial therapy with meropenem and vancomycin was initiated. The patient was transferred to the operating room where a lumbar drain was inserted and a revision procedure performed to identify and stop the CSF

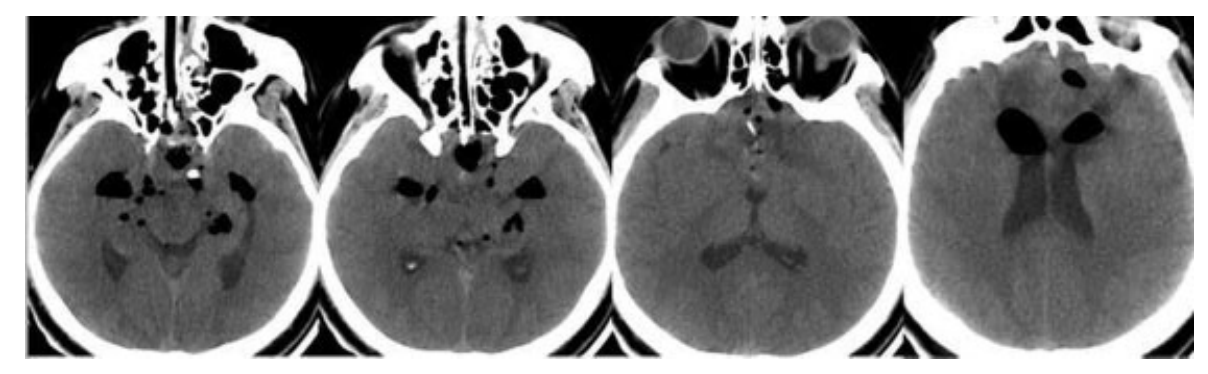

Fig. 2 Axial computed tomography brain scan immediately following tumor resection. 


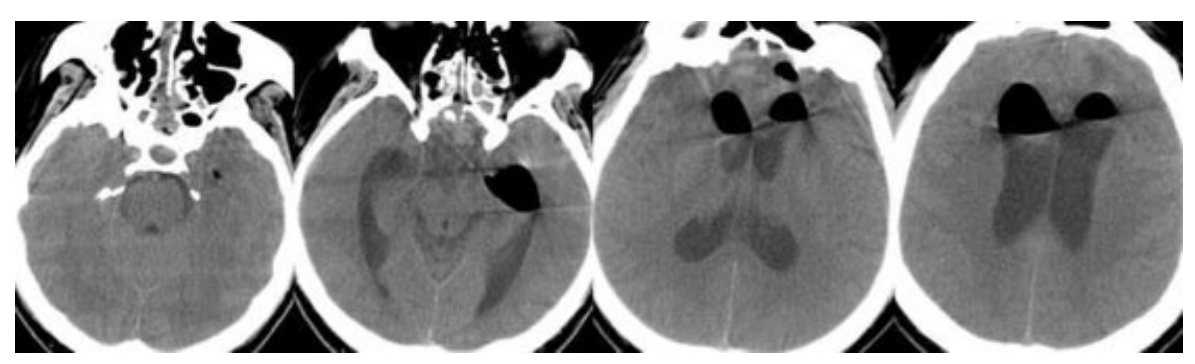

Fig. 3 Axial head computed tomography following deterioration of the patient's condition showing pneumocephalus.

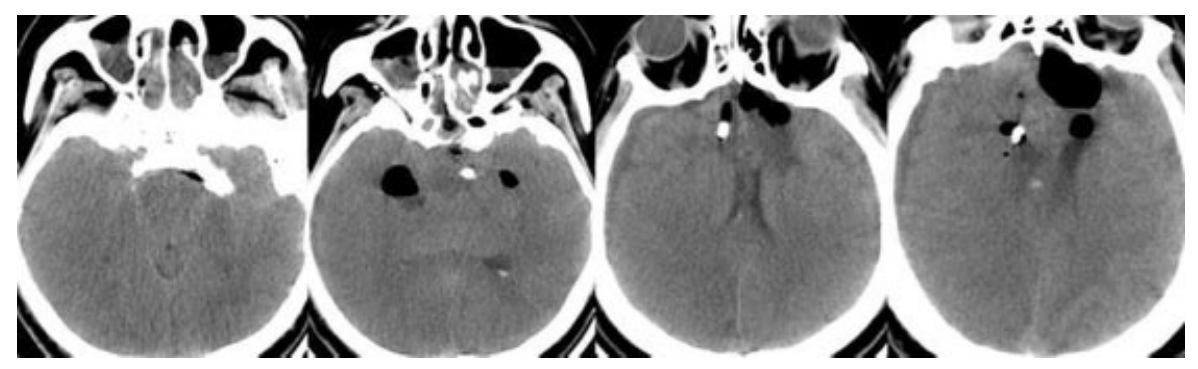

Fig. 4 Axial head computed tomography demonstrating malignant brain edema.

leak. The source of the leak was located in the capsule of the previously resected tumor and successfully sealed. The patient returned to the ICU sedated with propofol, intubated, and mechanically ventilated. A CT brain revealed generalized brain edema and obliterated basal cisterns ( - Fig. 4).

In response to these findings, lumbar drainage was discontinued and a ventricular drain with an ICP probe (Codman \& Shurtleff, Inc, Raynham, Massachusetts, United States) was inserted. ICP confirmed severe ICH $(25-35 \mathrm{~mm} \mathrm{Hg})$ that proved refractory to first-tier therapy with optimal bed positioning, propofol sedation, fentanyl analgesia, paralysis with intravenous pipecuronium $0.05 \mathrm{mg} / \mathrm{kg} /$ hour, $250 \mathrm{~mL}$ intravenous hyperosmolar solution (HyperHAES, Fresenius Kabi, Bad Homburg, Germany), and external ventricular drainage. CSF analysis confirmed the cause of ICH to be meningitis, with a CSF cell count of $14592 / \mu \mathrm{L}$, glucose of $0.2 \mathrm{mmol} / \mathrm{L}$, and lactate of $17 \mathrm{mmol} / \mathrm{L}$. Transcranial Doppler ultrasound (TCD) demonstrated increased mean velocity of middle cerebral artery (MCA) blood flow of up to $116 \mathrm{~cm} / \mathrm{second}$ with an associated maximal Lindegaard index of 3.4. The patient was hemodynamically unstable (BP: $80 / 40-85 / 45 \mathrm{~mm} \mathrm{Hg}$; HR: $50-60$ $\mathrm{bpm}$ ) and required a 0.03 to $0.05 \mu \mathrm{g} / \mathrm{kg} /$ minute norepineph- rine infusion to maintain a target mean arterial pressure of 95$110 \mathrm{~mm} \mathrm{Hg}$. C-reactive protein was elevated at $156 \mathrm{mg} / \mathrm{dL}$, significantly increased from $0.1 \mathrm{mg} / \mathrm{dL} 3$ days previously. A fever of $39^{\circ} \mathrm{C}$ developed. Polymerase chain reaction analysis revealed Acinetobacter baumannii in the CSF. Specific therapy with intravenous polymyxin E was started. Vancomycin was discontinued and meropenem continued while signs of meningitis in the form of elevated CSF cell count and CSF lactate and positive CSF microbiology persisted.

The decision was made to initiate therapeutic hypothermia for ICP control. The Thermogard XP temperature management system (Zoll, Chelmsford, Massachusetts, United States) was used with a heat-exchange catheter inserted into the right femoral vein. The target temperature range of 32.0 to $32.5^{\circ} \mathrm{C}$ was achieved in 3 hours. The ICP normalized from 25 to $35 \mathrm{~mm} \mathrm{Hg}$ to 10 to $15 \mathrm{~mm} \mathrm{Hg}$ over 1.5 hours. Attempts to rewarm the patient initially at 24- and then at 48hour intervals after induction of hypothermia failed due to ICH recurrence. Therapeutic hypothermia was thus continued for a total of 7 days when a trial of rewarming was successful and after which the patient was fully rewarmed over 48 hours. Repeat CT scanning (- Fig. 5) showed patent basal

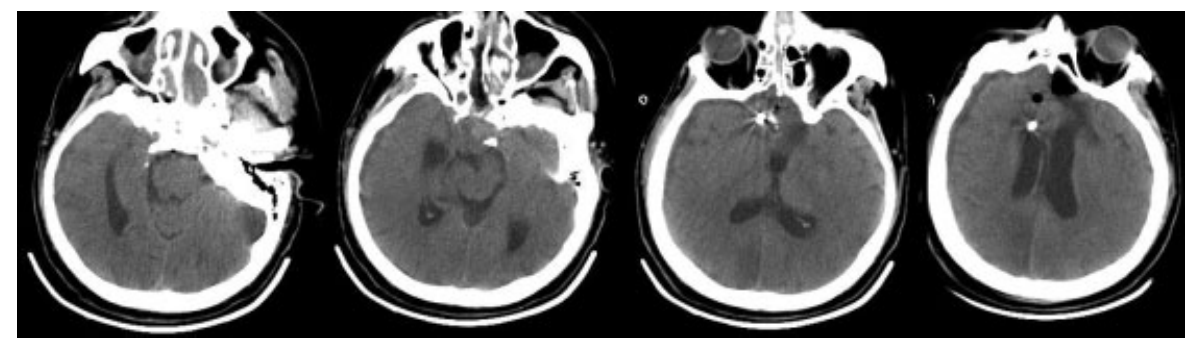

Fig. 5 Axial head computed tomography following cessation of cooling after 7 days. Mild hypothermia was continued until the patient was able to tolerate rewarming without refractory intracranial pressure spikes. In our case this was a full 7 days, at the limit of the maximum advised duration as recommended by the manufacturer. 
cisterns, resolving pneumocephalus, and reduced brain edema. ICP remained stable at 7 to $15 \mathrm{~mm} \mathrm{Hg}$, and the external ventricular drainage and ICP probe were removed. Mean MCA blood flow velocity reduced to 70 to $75 \mathrm{~cm} /$ second. CSF cell count decreased to $4 / \mu \mathrm{L}$ with correspondent reductions in lactate $(4.4 \mathrm{mmol} / \mathrm{L})$ and an increase in glucose to $5 \mathrm{mmol} / \mathrm{L}$, and meropenem was discontinued. Mechanical ventilation was continued, and the patient was hemodynamically stable. A trial off sedation revealed a GCS of 10/15 (E1V4M5).

Following this acute stage, the patient remained in the ICU for 62 days. During the initial 19 days, GCS was 12/15 (E1V5M6) after which time the patient opened his eyes spontaneously but was in a vegetative state that persisted for 33 days. Hydrocephalus developed, requiring ventriculoperitoneal shunting on the postoperative day 49 . The patient gradually became able to follow simple, repeated commands, corresponding to a minimally conscious state. MRI revealed extensive white matter changes and findings consistent with resolved intracranial infection and the consequences of brain edema and herniation. The patient was transferred to a rehabilitation unit in this condition.

\section{Discussion}

The patient described here presented with pituitary apoplexy secondary to intratumoral hemorrhage in a pilocytic astrocytoma, a rare entity in this region; pituitary adenomas are a much more common finding., ${ }^{2,3}$ Endoscopic transnasal transsphenoidal resection (ETTR) is now established as the approach of choice for tumors in this region, although postoperative meningitis is known to be an important and dangerous complication. ${ }^{4,5}$ Risk factors for meningitis following ETTR include intra- and postoperative CSF leak and the attendant risks of external lumbar or ventricular drainage. ${ }^{6}$ The extent to which truly sterile surgery through the nasal cavity is possible is also debatable. As a consequence of these factors, prompt recognition and treatment of postoperative meningitis is extremely important in cases treated with this approach. Early recognition of meningitis can be difficult, and a role for indirect detection including serial TCD measurements may exist. ${ }^{4}$

Diagnosis in this case was relatively straightforward with CSF analysis unambiguous for bacterial infection. Our patient was treated optimally, with broad-spectrum antibiotics administered until a specific organism was cultured, and the CSF leak identified and closed surgically in a timely fashion.

The postoperative period was unfortunately complicated by severe ICH, refractory to first-tier therapies and necessitating consideration of second-tier options, namely hyperventilation, barbiturate coma, decompressive hemicraniectomy, and therapeutic hypothermia. ${ }^{1}$

Although decompressive hemicraniectomy considerably enlarges the volume available for brain expansion and is effective in reducing ICP, ${ }^{7}$ its effect on neurologic outcomes remains a contentious issue. ${ }^{8-10}$ Because hemicraniectomy necessitates duraplasty, this option was contraindicated due to fulminant meningitis. The foreign material required by this procedure would immediately become a new infective nidus.
Aggressive hyperventilation (partial pressure of carbon dioxide $\left[\mathrm{PCO}_{2}\right]<28 \mathrm{~mm} \mathrm{Hg}$ ) is no longer used in neurocritical care practice due to the recognized risk of vasoconstriction and consequent brain ischemia. ${ }^{11}$ In any event, our patient could not be hyperventilated because the increased cerebral blood flow velocities already established with TCD meant the risk of vasoconstriction, and ischemia precluded further safe reduction in $\mathrm{PCO}_{2}$.

The use of barbiturate coma for ICP control was the subject of a comprehensive Cochrane review that concluded this method should be avoided. ${ }^{12}$ This recommendation is grounded in the observation that barbiturates can depress hemodynamics and worsen cerebral perfusion and neurologic outcomes. In the present case, in a patient already requiring inotropes to maintain hemodynamic stability, barbiturates were deemed not to be an option.

Mild hypothermia has been shown to be an effective method of ICP control. ${ }^{13-16}$ It is thought that the neuroprotective effect of hypothermia is related to a beneficial reduction in brain metabolism, lessening edema and free radical production and leading to less secondary brain injury. ${ }^{7}$ Hypothermia may additionally inhibit some of the deleterious processes specific to bacterial meningitis, many of which have been shown to be temperature dependent. ${ }^{17,18}$

In the situation described here, hypothermia remained the only second-tier therapy available due to the contraindications to other measures described earlier. The Thermogard XP system rapidly decreased core body temperature in our patient and allowed ICP to be kept below $20 \mathrm{~mm} \mathrm{Hg}$. Despite this, our patient did not develop shivering or require additional sedation or paralysis. This is in line with support in the literature for a decreased incidence of shivering during Thermogard system use when compared with other methods of attaining therapeutic hypothermia. ${ }^{19-22}$

Optimal duration of therapeutic hypothermia is yet to be defined, ${ }^{22}$ with strong guidelines existing only for hypothermia following cardiac arrest and in neonates with hypoxicischemic encephalopathy, $13,23,24$ suggesting that these groups should be cooled for 24 hours. Strong evidence in other patient groups is lacking. It is extremely important to strike a balance between the risk of ICH recurrence in early rewarming and the risk of complications developing from prolonged hypothermia, either of which can worsen outcomes. ${ }^{15,25,26}$

Our attempts to terminate hypothermia as soon as possible and to rewarm the patient at 24- and subsequently at 48-hour intervals were unsuccessful until day 7 of hypothermia due to immediate ICH recurrence, highlighting differences in the pathogenic mechanisms of cerebral edema after cardiac arrest and in ongoing meningitis. It is apparent that the mechanisms resulting in brain edema in patients with meningitis remain in effect for several days, whereas in post-arrest patients the influence of these factors recedes earlier. It is noteworthy that 7 days of catheter-mediated therapeutic hypothermia did not lead to any complications in this case. Seven days is the maximum advised duration of therapy as recommended by the manufacturers. To minimize complications, we used a therapeutic dose of low molecular weight 
heparin throughout cooling and supplemented this with daily ultrasound examinations of the lower extremities, as well as careful aseptic technique on insertion of the catheter and meticulous care of the puncture site. It seems reasonable to suggest that hypothermia initiated for ICP control in patients secondary to meningitis should be continued for at least 72 hours, with this time period extended further if clinically warranted. The safety and efficacy of prolonged use of the Thermogard system is also supported by this case.

Sadly, the outcome in this case was less than optimal, with the patient discharged in a minimally conscious state. The risk of such unfavorable neurologic outcomes is always high in situations when first-tier therapy is ineffective and secondtier options are limited. The risk of such outcomes cannot be a contraindication for the use of hypothermia in such patients, although its judicious use should be discussed within the treating multidisciplinary team and with patients' families.

Experiences such as these mandate the exploration of further strategies combining hypothermia with other approaches to improve outcomes. Such combinations have been fruitful in studies of malignant stroke, where hypothermia combined with decompressive hemicraniectomy was proven effective. ${ }^{27-29}$ Another successful example of such an approach is a combination of hypothermia and cerebral thrombolysis. ${ }^{30}$ In patients with sellar region tumors, optimal polyhormonal therapy in conjunction with hypothermia may improve outcomes. ${ }^{4,31}$

\section{Conclusion}

The case presented here outlines the lifesaving role of therapeutic hypothermia when other second-tier ICP management options are contraindicated or otherwise unavailable. The patient ultimately required 7 days of cooling with the Thermogard XP system, demonstrating its safety and efficacy during prolonged use. Although the neurologic outcome in this case was not optimal, hypothermia was undoubtedly lifesaving, and its combination with other neuroprotective therapies may result in improved outcomes in cases like these.

\section{Conflict of Interest}

Ronald Melieste is vice president of the International Development and Temperature Management Division Europe, Middle East, Africa and Latin America of Zoll Medical Corporation, the manufacturer of the Thermogard cooling system. The other authors have nothing to disclose.

\section{References}

1 Dennis LJ, Mayer SA. Diagnosis and management of increased intracranial pressure. Neurol India 2001;49(Suppl 1):S37-S50

2 Goldberg PA, Inzucchi SE. Critical issues in endocrinology. Clin Chest Med 2003;24(4):583-606, vi

3 Shimon I, Melmed S. Management of pituitary tumors. Ann Intern Med 1998;129(6):472-483
4 Popugaev KA, Savin IA, Lubnin AU, et al. Unusual cause of cerebral vasospasm after pituitary surgery. Neurol Sci 2011;32(4): 673-680

5 Kassam AB, Prevedello DM, Carrau RL, et al. Endoscopic endonasal skull base surgery: analysis of complications in the authors' initial 800 patients. J Neurosurg 2011;114(6):1544-1568

6 Nishioka H, Haraoka J, Ikeda Y. Risk factors of cerebrospinal fluid rhinorrhea following transsphenoidal surgery. Acta Neurochir (Wien) 2005;147(11):1163-1166; discussion 1166

7 Schreckinger M, Marion DW. Contemporary management of traumatic intracranial hypertension: is there a role for therapeutic hypothermia? Neurocrit Care 2009;11(3):427-436

8 Cooper DJ, Rosenfeld JV, Murray L, et al; DECRA Trial Investigators Australian and New Zealand Intensive Care Society Clinical Trials Group. Decompressive craniectomy in diffuse traumatic brain injury. N Engl J Med 2011;364(16):1493-1502

9 Marion DW. Decompressive craniectomy in diffuse traumatic brain injury. Lancet Neurol 2011;10(6):497-498

10 Meier U, Ahmadi S, Killeen T, Al-Zain FT, Lemcke J. Long-term outcomes following decompressive craniectomy for severe head injury. Acta Neurochir Suppl (Wien) 2008;102:29-31

11 Stocchetti N, Maas AI, Chieregato A, van der Plas AA. Hyperventilation in head injury: a review. Chest 2005;127(5):1812-1827

12 Roberts I. Barbiturates for acute traumatic brain injury. [update in Cochrane Database Syst Rev 2012;12:CD000033]. Cochrane Database Syst Rev 2001;1:CD000033

13 Arrich J; European Resuscitation Council Hypothermia After Cardiac Arrest Registry Study Group. Clinical application of mild therapeutic hypothermia after cardiac arrest. Crit Care Med 2007;35(4):1041-1047

14 Hemmen TM, Lyden PD. Hypothermia after acute ischemic stroke.J Neurotrauma 2009;26(3):387-391

15 Polderman KH. Application of therapeutic hypothermia in the ICU: opportunities and pitfalls of a promising treatment modality. Part 1: Indications and evidence. Intensive Care Med 2004;30(4): 556-575

16 Badjatia N. Hyperthermia and fever control in brain injury. Crit Care Med 2009;37 (7, Suppl):S250-S257

17 Lepur D, Kutleša M, Baršić B. Induced hypothermia in adult community-acquired bacterial meningitis-more than just a possibility? J Infect 2011;62(2):172-177

18 Polderman KH. Mechanisms of action, physiological effects, and complications of hypothermia. Crit Care Med 2009;37(7, Suppl): S186-S202

19 Aiyagari V, Diringer MN. CoolGard/Cool Line catheter system. Neurocrit Care 2004;1(2):209-211

20 Hinz J, Rosmus M, Popov A, Moerer O, Frerichs I, Quintel M. Effectiveness of an intravascular cooling method compared with a conventional cooling technique in neurologic patients. J Neurosurg Anesthesiol 2007;19(2):130-135

21 Schmutzhard E, Engelhardt K, Beer R, et al. Safety and efficacy of a novel intravascular cooling device to control body temperature in neurologic intensive care patients: a prospective pilot study. Crit Care Med 2002;30(11):2481-2488

22 Schwab S, Georgiadis D, Berrouschot J, Schellinger PD, Graffagnino C, Mayer SA. Feasibility and safety of moderate hypothermia after massive hemispheric infarction. Stroke 2001;32(9):2033-2035

23 Shankaran S. Neonatal encephalopathy: treatment with hypothermia. J Neurotrauma 2009;26(3):437-443

24 Jacobs SE, Berg M, Hunt R, Tarnow-Mordi WO, Inder TE, Davis PG. Cooling for newborns with hypoxic ischaemic encephalopathy. Cochrane Database Syst Rev 2013;1:CD003311

25 Hayashi N. Management of pitfalls for the successful clinical use of hypothermia treatment. J Neurotrauma 2009;26(3):445-453

26 Kilpatrick MM, Lowry DW, Firlik AD, Yonas H, Marion DW. Hyperthermia in the neurosurgical intensive care unit. Neurosurgery 2000;47(4):850-855; discussion 855-856 
27 Cruz-Flores S, Berge E, Whittle IR. Surgical decompression for cerebral oedema in acute ischaemic stroke. Cochrane Database Syst Rev 2012;1:CD003435

28 Georgiadis D, Schwarz S, Aschoff A, Schwab S. Hemicraniectomy and moderate hypothermia in patients with severe ischemic stroke. Stroke 2002;33(6):1584-1588

29 Els T, Oehm E, Voigt S, Klisch J, Hetzel A, Kassubek J. Safety and therapeutical benefit of hemicraniectomy combined with mild hypothermia in comparison with hemicraniectomy alone in patients with malignant ischemic stroke. Cerebrovasc Dis 2006; 21(1-2):79-85

30 Krieger DW, De Georgia MA, Abou-Chebl A, et al. Cooling for acute ischemic brain damage (cool aid): an open pilot study of induced hypothermia in acute ischemic stroke. Stroke 2001;32(8):1847-1854

31 Popugaev K, Savin I, Goriachev A, et al. Absence of acute phase of neuroendocrine response to critical illness state in sellar region tumour patients. [abstract]. Neurocrit Care 2010; 13 (1, Suppl): 160 